\title{
COMMENTARY
}

\section{Down but not out: myocardial depression in sepsis}

\author{
Anthony S McLean* \\ See related research by Weng et al., http://ccforum.com/content/16/3/R71
}

\begin{abstract}
Myocardial depression in septic patients is well recognized yet still poorly understood. The prognostic significance in terms of overall mortality when it is identified, remains in dispute. Parameters of left ventricular function measured by tissue Doppler imaging may assist in resolving whether dysfunction identified early in the course of sepsis is a good prognostic sign.
\end{abstract}

Myocardial impairment resulting from systemic sepsis has been recognized for more than a quarter of a century, yet much about the phenomenon remains a mystery. The incidence of myocardial depression in the critically ill patient with sepsis is uncertain because of the imprecision with which myocardial depression is described, the definition of sepsis, and the fact that published studies display marked heterogeneity in patient selection and methods of evaluation. For example, earlier studies included variable populations of patients and relied on radionuclide or pulmonary artery catheter methods, whereas techniques such as tissue Doppler imaging have been around for only the last decade. Depression of myocardial function in septic patients is likely to be more common than previous thought because echocardiography is now more readily available and used by critical care physicians experienced in using it. Weng and colleagues [1], in measuring echocardiographic, clinical, and laboratory variables in 61 patients with septic shock, found that left ventricular tissue Doppler parameters demonstrated potentially useful prognostic information. In particular, the peak systolic velocity measured at the mitral annulus (Sa), a measure of the long-axis systolic motion of the left ventricle, was significantly lower in survivors than non-survivors. Using a cutoff value of $9 \mathrm{~cm} /$ second predicted 90 -day mortality with a sensitivity

*Correspondence: anthony.mclean@sydney.edu.au

Department of Intensive Care Medicine, Nepean Hospital, University of Sydney, PO Box 63, Penrith NSW 2751, Australia and a specificity of $75 \%$ and $86 \%$, respectively. Left ventricular ejection fraction measurements between survivors and non-survivors were not significantly different. This work is the latest in a series of studies examining the pathophysiology of sepsis-induced cardiomyopathy and, more specifically, its prognostic significance.

Earlier work using radionuclide and pulmonary artery catheter studies indicated that dilatation of the left ventricle was associated with a lower mortality in patients with sepsis [2]. Later studies using echo Doppler techniques did not confirm the relationship between left ventricular dilatation and improved outcome from sepsis but did confirm the relationship between left ventricular dysfunction and outcome. Vieillard-Baron and colleagues [3] found that $60 \%$ of their patients with sepsis experienced underlying left ventricular dysfunction as a result of sepsis when evaluated by transesophageal echocardiographic examination. The assumption that septic cardiomyopathy is somehow beneficial and indicates a better patient outcome should be treated with caution. This is an attractive hypothesis, but the focus may be incorrect. The contrary emphasis could be that the hyperkinetic state present in many patients with sepsis indicates profound vasoplegia and this is responsible for the increased mortality in this group [4]. Also, the clinician needs to consider more than left ventricular systolic function as left ventricular diastolic function and right heart function can also be affected in sepsis, either independently or collectively [5]. Even when the more recent sophisticated echo Doppler techniques are applied, there is disagreement regarding the prognostic or potentially protective value of cardiac depression. In a study of only 21 patients, E/Ea (early diastolic transmitral velocity/early mitral annular diastolic velocity ratio), but not $\mathrm{Sa}$, was an independent predictor of mortality in septic shock [6]. In a larger and more recent study involving 262 patients with severe sepsis, the presence of systolic dysfunction, diastolic dysfunction, or both was associated with a higher mortality [7].

The actual cause of the cardiac dysfunction found in many patients with severe sepsis is still uncertain and multiple etiologies are proposed. Cytokines have been favorite candidates over the years; a circulating myocardial depressant factor was first postulated by Wiggers 
[8] in 1947 and was advanced further by Parillo and colleagues [9] in 1985. Complement molecules, nitric oxide, cellular adhesion molecules, disordered intracellular energetics, and abnormalities in intracellular calcium fluxes are some of the more recently postulated causes [10].

Considerable uncertainty still surrounds the incidence, etiology, assessment, and prognostic significance of septic cardiomyopathy, and the findings by Weng and colleagues should be observed in this light. It is unlikely that a single measurement such as Sa will prove to be a robust prognostic marker, particularly since the range for this parameter in the healthy population is so broad [11]. It may be that recently developed echo Doppler techniques will provide a more accurate set of methods of evaluating cardiac dysfunction in severe sepsis. Recent developments, including velocity vector tracking and fourdimensional echocardiography, open a window on ventricular rotation during systole, a significant contributor to cardiac output. In the past, echo Doppler techniques - which, apart from magnetic resonance imaging, are the best tools available for assessing ventricular function - have not taken into account the contribution of twisting and torsion of the ventricle, estimated to account for $40 \%$ of cardiac output. Tissue Doppler imaging has enhanced our understanding of left ventricular function, and the finding that Sa may be an important prognostic marker in septic cardiomyopathy is a stimulus to more closely examine myocardial reaction to the insult. The cardiac sequelae of severe sepsis can be severe, and bedside echocardiographic assessment is essential to the further understanding of this most intriguing condition.

Abbreviation

Sa, systolic velocity measured at the mitral annulus.
Competing interests

The author declares that he has no competing interests.

Published: 27 June 2012

\section{References}

1. Weng L, Liu YT, Du B, Zhou JF, Guo XX, Peng JM, Hu XY, Zhang SY, Fang Q, Zhu WL: The Prognostic value of left ventricular systolic function as measured by tissue Doppler imaging in septic shock. Crit Care 2012, 16:R71.

2. Parker MM, Suffredini AF, Natanson C, Ognibene FP, Shelhamer JH, Parrillo JE: Responses of left ventricular function in survivors and nonsurvivors of septic shock. J Crit Care 1989, 4:19-25.

3. Vieillard-Baron A, Caille V, Charron C, Belliard G, Page B, Jardin F: Actual incidence of global left ventricular hypokinesia in adult septic shock. Crit Care Med 2008, 36:1701-1706.

4. Vieillard-Baron A: Septic cardimyopathy. Ann Intensive Care 2011, 1:6.

5. Hunter JD, Doddi M: Sepsis and the heart. Brit J Anaesthesia 2010, 104:3-11.

6. Sturgess DJ, Marwick TH, Joyce C, Jenkins C, Jones M, Masci P, Stewart D, Venkatesh B: Prediction of hospital outcome in septic shock: a prospective comparison of tissue Doppler and cardiac biomarkers. Crit Care 2010, 14:R44.

7. Landesberg G, Gilon D, Meroz Y, Georgieva M, Levin PD, Goodman S, Avidan A, Beeri R, Weissman C, Jaffe AS, Sprung CL: Diastolic dysfunction and mortality in septic shock. Eur Heart J 2012, 33:895-903.

8. Wiggers CJ: Myocardial depressant factor in shock. A survey of cardiodynamic studies. Am Heart J 1947, 33:633-650.

9. Parillo JE, Burch C, Shelhamer JH, Parker MM, Natanson C, Schuette W: A circulating myocardial depressant substance in humans with septic shock. Septic patients with a reduced ejection fraction have a circulating factor that depresses in vitro myocardial cell performance. J Clin Invest 1985, 76:1539-1553.

10. Flynn A, Mani BC, Mather PJ: Sepsis-induced cardiomyopathy:a review of pathophysiologic mechanisms. Heart Fail Rev 2010, 15:605-611.

11. Chahal NS, Lim TK, Jain P, Chambers JC, Kooner JS, Senior R: Normative reference values for the tissue Doppler imaging parameters of left ventricular function: a population-based study. Eur J Echocardiogr 2010, 11:51-56.

doi:10.1186/cc11367

Cite this article as: McLean AS: Down but not out: myocardial depression in sepsis. Critical Care 2012, 16:132. 